\title{
ANÁLISE DO USO DO APLICATIVO TELEGRAM PARA O CONTROLE DE PROCESSOS DE MANUTENÇÃO DE AERONAVES
}

\section{ANALYSIS OF THE USE OF TELEGRAM APPLICATION TO CONTROL AIRCRAFT MAINTENANCE PROCESSES}

\author{
Paulo George Miranda Martinsa \\ Rogério Aparecido Sá Ramalho \\ Helton Luiz dos Santos Graciano ${ }^{\circ}$
}

\begin{abstract}
RESUMO
Introdução: Com o desenvolvimento das novas tecnologias de informação e comunicação o compartilhamento instantâneo de dados e informações possibilitaram mudanças significativas no comportamento das organizações. No âmbito da manutenção de aeronaves, o emprego dessas tecnologias ganha novos contornos com a utilização de aplicativos de mensagens no gerenciamento de suas atividades e controle de seus processos, permitindo dimensionar com mais eficiência a aplicação de recursos. Objetivo: $O$ objetivo central desse trabalho consiste em analisar a utilização do aplicativo Telegram no gerenciamento de atividades relacionadas aos processos da área de Controle de Qualidade de uma empresa de manutenção de aeronaves e seus reflexos no âmbito da Gestão do Conhecimento. Metodologia: Caracteriza-se como uma pesquisa-ação que buscou auxiliar na identificação de diretrizes e análise de soluções para a melhoria contínua de processos da área de Controle de Qualidade. Resultados: É apresentada uma análise do uso do aplicativo Telegram para o aperfeiçoamento de processos de manutenção, destacando a relevância do uso de novas abordagens tecnológicas para 0 atendimento de demandas relacionadas à Gestão do Conhecimento em ambientes corporativos. Conclusões: A partir da análise realizada constatou-se que o uso do aplicativo Telegram contribuiu para uma maior sinergia entre as equipes de trabalho ao compartilhar informações essenciais ao processo, tornando 0 atendimento e demais demandas processos mais ágeis e sincronizadas com as propostas de segurança, qualidade e confiabilidade, favorecendo melhoras significativas nos processos de Gestão do Conhecimento em ambientes corporativos.
\end{abstract}

\footnotetext{
a Mestre em Ciência da Informação pelo Programa de Pós-Graduação em Ciência da Informação da Universidade Federal de São Carlos (UFSCar). E-mail: pgeorgemm22@gmail.com

b Doutorado em Ciência da Informação pela Universidade Estadual Paulista (UNESP). Docente do Programa de Pós-Graduação em Ciência da Informação da Universidade Federal de São Carlos (UFSCar).E-mail: ramalho@ufscar.br

c Mestrando em Ciência da Informação pelo Programa de Pós-Graduação em Ciência da Informação da Universidade Federal de São Carlos (UFSCar). E-mail: helton.graciano@gmail.com
} 
Paulo George Miranda Martins, Rogério Aparecido Sá Ramalho, Helton Luiz dos Santos Graciano

Análise do uso do aplicativo telegram para o controle de processos de manutenção de aeronaves

Descritores: Tecnologias da informação. Gerenciamento de Processos. Manutenção de Aeronaves. Inovação. Telegram.

\section{INTRODUÇÃO}

Com o desenvolvimento das novas Tecnologias de Informação e Comunicação (TIC), um dos principais objetivos da área de Ciência da Informação é proporcionar subsídios teóricos e práticos acerca das tecnologias emergentes, possibilitando o desenvolvimento de ferramentas e métodos eficazes no compartilhamento dos mais diversos recursos, sobretudo em ambientes dinâmicos como a Web.

A quantidade de dados e informações disponíveis no contexto contemporâneo tem provocado uma grande transformação na forma como os recursos informacionais são utilizados, principalmente, dado a sua variedade e formato. Tais implicações traz uma nova perspectiva na forma como as organizações lidam com grandes volumes de informações e a maneira como as mesmas podem ser utilizadas para tomada de decisão a partir das relações entre bancos de dados estruturados e outros dados correlacionados.

O ambiente Web constitui-se como uma das mais ricas fontes de informação (RAMALHO, 2016) e o uso adequado de tecnologias com grande poder de compartilhamento permite que a acessibilidade de conteúdos e a troca de informações seja potencializada, uma vez que tais informações podem ser recuperadas e utilizadas de forma mais rápida (SAEGER et al., 2016).

Nesse contexto, a informação constitui um recurso essencial para as tomadas de decisão em uma organização e a utilização das TIC em ambientes corporativos possibilita novas perspectivas no âmbito do gerenciamento informacional e de processos, favorecendo a transmissão de dados de forma maciça, permitindo a recuperação e o compartilhamento de conteúdo.

Para os autores Saeger et al. (2016), as informações coletadas e analisadas adequadamente podem ser utilizadas como um recurso estratégico nas organizações, permitindo que melhorias em seus processos sejam implantadas de forma eficiente.

Dessa maneira, o emprego das tecnologias de informação e comunicação 
Paulo George Miranda Martins, Rogério Aparecido Sá Ramalho, Helton Luiz dos Santos Graciano

Análise do uso do aplicativo telegram para o controle de processos de manutenção de aeronaves

assume um papel importante na comunicação no âmbito corporativos, podendo ser utilizados como ferramentas para viabilizar uma comunicação direta e sinérgica e, a manipulação e a reorganização de demandas nos mais diversos setores da organização, fato esse proporcionado pelo crescente uso de dispositivos móveis como tablets e smartphones.

No âmbito aeronáutico, tema no qual esse trabalho versa, o uso dessas tecnologias permite um melhor uso das informações e um controle mais eficaz dos processos inerentes à manutenção de aeronaves uma vez que, a alta competitividade nesse setor requer que as empresas adotem planos estratégicos mais consistentes e eficazes no atendimento de suas demandas operacionais (CRUZ, 2016).

Nessa perspectiva, o objetivo central dessa pesquisa foi analisar a utilização do aplicativo Telegram no gerenciamento de atividades relacionadas aos processos da área de Controle de Qualidade de uma empresa de manutenção de aeronaves e seus reflexos no âmbito da Gestão do Conhecimento.

Quanto aos procedimentos metodológicos, utilizou-se o método de pesquisa-ação uma vez que se objetiva identificar os principais fatores relacionados a um determinado problema e auxilia no desenvolvimento de diretrizes e soluções práticas a serem empregados (McKAY; MARSHALL, 2001).

Segundo Thiollent (2005) a metodologia de pesquisa-ação favorece a identificação das problemáticas inerentes ao contexto abordado e contribui para o desenvolvimento de ações efetivas e duradouras. Caracteriza-se, também, como pesquisa de natureza aplicada, uma vez que "[...] objetiva gerar conhecimentos para aplicação prática, dirigidos à solução de problemas específicos" (SILVEIRA; CÓRDOVA, 2009, p.35).

Aborda-se os aspectos de utilização das tecnologias de informação e comunicação no âmbito de prestação de serviços, como o setor de manutenção de aeronaves, que dado ao grande volume de informações se utiliza de tais tecnologias no auxílio de tomadas de decisão em seus processos. Destaca-se o emprego das TIC no setor e sua contribuição efetiva na padronização dos atendimentos prestados pelo Controle da Qualidade, que passa a planejar e 
Paulo George Miranda Martins, Rogério Aparecido Sá Ramalho, Helton Luiz dos Santos Graciano

Análise do uso do aplicativo telegram para o controle de processos de manutenção de aeronaves

controlar de forma eficiente o emprego de seus recursos, além de promover uma melhor sinergia entre as diversas áreas envolvidas no processo de manutenção.

\section{TECNOLOGIAS DA INFORMAÇÃO E GESTÃO DE PROCESSOS}

O rápido desenvolvimento da Internet e a crescente disposição e compartilhamento de dados e informações em larga escala tem acarretado uma mudança na forma como lidamos com os mais variados recursos e como enxergamos o ambiente Web. A influência de novas tendências de pesquisas envolvendo Linked Data; Data Science e Web Semântica, que em suma possibilita a interligação e agregação semântica de recursos em níveis satisfatórios, à demanda por tecnologias com capacidade de trabalhar com grandes volumes de dados no contexto de Big Data (SOUSA; MARTINS; RAMALHO, 2018) são cada vez mais necessárias sob a nova perspectiva de mercado que lida com análise e controle de uma enorme variedade de informações.

Nesse contexto a área de Ciência da Informação tem contribuído significativamente com estudos direcionados ao desenvolvimento de novos métodos e modelos de representação, transmissão, armazenamento e criação de tecnologias capazes de possibilitar um compartilhamento eficaz de informações. Assim, o uso dessas tecnologias propicia a colaboração mútua entre diversas organizações, permitindo que todos possam contribuir com conhecimentos únicos de forma que as informações sejam reutilizadas e recombinadas (BAKER et al., 2011, não paginado, tradução nossa).

Com o rápido desenvolvimento de tecnologias para atender tais demandas, outros aportes tecnológicos inseridos no bojo das TIC podem ser utilizados para favorecer uma relação mais estreita entre os diversos setores das organizações, criando oportunidades, crescimento em seu escopo de atuação e aumento da competitividade já que essas tecnologias permitem identificar, através da análise de dados e informações, pontos de melhoria nos processos e redução de custos (FAGUNDES, 2019).

São através dessas tecnologias que os aplicativos de mensagens amplamente utilizados pelo público em geral, como o Telegram, podem 
Paulo George Miranda Martins, Rogério Aparecido Sá Ramalho, Helton Luiz dos Santos Graciano

Análise do uso do aplicativo telegram para o controle de processos de manutenção de aeronaves

potencializar o fluxo informacional e prover uma melhor interação entre grupos e setores distintos da sociedade, permitido que informações e dados sejam amplamente controlados e manipulados.

Para Feijo, Silva e Benetti (2018), tais tecnologias estão inseridas em um contexto de grandes transformações sociais e tecnológicas e o compartilhamento instantâneo de dados e informações permite que qualquer tipo de recurso possa ser recuperado e utilizado para os mais diversos fins.

O uso de aplicativos de mensagens no gerenciamento de processos é uma realidade cada vez mais presente em setores em que as tomadas de decisão necessitam ocorrer de forma rápida e dentro das diretrizes prédeterminadas no planejamento estratégico. Associadas as demais práticas do sistema produtivo, essas tecnologias contribuem de forma significativa para o redesenho na forma de atendimento das demandas, minimizando perdas e potencializando oportunidade de ganhos em áreas de apoio que são determinantes para o fluxo sistêmico no âmbito da manutenção.

Borges, Lessa e Oliveira (2014) destacam que, essa nova frente de atuação requer das organizações a capacidade de manterem-se informadas e ao mesmo tempo estabelecerem estratégias de comunicação eficazes com diferentes atores, como: o público para o qual suas atividades são direcionadas, seus parceiros, colaboradores, o poder público e, também, à sociedade como um todo, buscando apoio e visibilidade de seus produtos e serviços.

A informação é o principal insumo para o desenvolvimento em uma organização e a compreensão de sua importância deve estar presente em todos os setores da empresa no qual estabelece suas tomadas de decisão a partir de informações coletadas e selecionadas, sendo que sua escolha é um processo racional embasado em dados sobre as metas predefinidas, os resultados a serem obtidos e os valores gerados desses resultados (CHOO, 1996).

Metas e objetivos são definidos primeiro, e quando os participantes encontram problemas na busca desses objetivos, uma busca por informações sobre alternativas e consequências ocorre seguida pela avaliação dos resultados de acordo com os objetivos e preferências (CHOO, 1996, p. 322).

Tal afirmação justifica-se pelo pressuposto de que o conhecimento gerado a partir das informações e dados convergentes de diversas fontes constitui um 
Paulo George Miranda Martins, Rogério Aparecido Sá Ramalho, Helton Luiz dos Santos Graciano

Análise do uso do aplicativo telegram para o controle de processos de manutenção de aeronaves

aspecto importante que "[...] garantirá vantagem competitiva em cenários de inovação e competição e sua sistematização é algo essencial para o uso em processos de trabalho e nos momentos de tomada de decisão" (SOUZA; AGANETTE, 2018, p. 5130).

Segundo Canaday (2018), no segmento produtivo é cada vez mais comum o uso de ferramentas tecnológicas desenvolvidas no contexto das TIC, no qual novas funcionalidades são incorporadas a partir dos avanços da Inteligência Artificial (IA) para o controle assertivo dos processos.

No âmbito da manutenção de aeronaves, gerir de forma eficiente as atividades desempenhadas, estabelecer metas, traçar estratégias e estipular prazos são essenciais para o sucesso de qualquer projeto e, o uso de informações corretas faz toda diferença para as tomadas de decisão uma vez que proporcionam vantagem competitiva ao direcionar as ações preventivas ou corretivas em pontos específicos do processo.

Nesse contexto, a qualidade das informações é fundamental para as tomadas de decisão, pois auxiliam os gestores na avaliação de problemas e oportunidades, priorizando mudanças nas atividades e o redesenho de sistemas, processos e práticas (ALBRECHT, 1999).

O uso eficiente das informações que permeiam a área de manutenção é um fator relevante para o planejamento estratégico entre as equipes e torna-se essencial para um resultado satisfatório uma vez que a eficácia na execução das atividades planejadas requer um compartilhamento sinérgico de informações com as demais áreas de apoio (MACHADO; URBINA; ELLER, 2015).

Para Cruz (2016), a eficácia nos processos na manutenção depende do cumprimento das decisões estratégicas estabelecidas pela organização, cujo fluxo constante de informação possibilita o cumprimento de prazos, a qualidade dos serviços e a capacidade de resiliência frente às mudanças que podem ocorrer.

Administrar de forma eficiente todos os objetivos propostos, transformando-os em ações efetivas na organização, é uma missão primordial para garantir que o planejamento estratégico, a organização e o controle de todos os esforços empregados na manutenção atinjam seus objetivos de 
Paulo George Miranda Martins, Rogério Aparecido Sá Ramalho, Helton Luiz dos Santos Graciano

Análise do uso do aplicativo telegram para o controle de processos de manutenção de aeronaves

maneira efetiva, garantindo com isso a competitividade, qualidade e uso adequado dos recursos (CHIAVENATO, 2003; CORREIA, 2012).

Nessa perspectiva, surge uma nova tendência de uso das TIC a partir da utilização do aplicativo de mensagens Telegram no setor de manutenção aeronáutica, no qual novas formas de compartilhamento de informações permite gerenciar recursos e o tempo empregado nas atividades de manutenção, possibilitando que todos os profissionais da organização possam partilhar do mesmo propósito, influenciando a produtividade e os bons resultados, cujo objetivo final é a utilização das melhores práticas para se alcançar o estado de excelência.

\section{O USO DO TELEGRAM NO CONTROLE DE PROCESSOS}

A qualidade na fabricação de produtos e prestação de serviços ganha cada vez mais força a partir da utilização das novas tecnologias de comunicação e informação empregadas para gerenciar os mais diversos processos na cadeia produtiva, sistematizando de forma eficiente o uso dos recursos disponíveis, evitando os altos custos resultantes dos erros decorrentes de manipulações e interpretações equivocadas de informações.

Com a popularização de smartphones, aplicativos de mensagens têm sido cada vez utilizados para o compartilhamento instantâneo de conteúdos informacionais abrangendo um grupo cada vez maior de usuários. Assim, a utilização de tais tecnologias possibilita uma redução significativa nos custos operacionais de uma organização, uma vez que o emprego de tempo, recursos humanos, financeiros, dentre outros podem ser dimensionados de forma eficaz no aproveitamento dos mais diversos recursos que são destinados ao controle e coordenação da manutenção, tornando os processos mais ágeis e integrados entre si (MACHADO; URBINA; ELLER, 2015).

Segundo Silveira et al. (2006), tal percepção é ratificada pelo fato da rápida difusão e uso dessas tecnologias nos mais variados setores, favorecendo a integração de diferentes áreas, a circulação e troca de dados, a disseminação de informações nas redes eletrônicas e a geração de novos conhecimentos.

$\mathrm{Na}$ contemporaneidade, uma das tecnologias emergentes utilizadas para 
Paulo George Miranda Martins, Rogério Aparecido Sá Ramalho, Helton Luiz dos Santos Graciano

Análise do uso do aplicativo telegram para o controle de processos de manutenção de aeronaves

a comunicação instantânea é o aplicativo Telegram que, nessa pesquisa é abordado pela sua aplicação no gerenciamento de recursos na área do Controle da Qualidade (CQ) de uma unidade de Maitenance, Repair and Overhaul (MRO) ou centro de manutenção de aeronaves e componentes de uma empresa aérea, que o utiliza para o controle de atendimento das tarefas de manutenção.

Lançado em 2013, o Telegram surge com a proposta de possibilitar uma comunicação rápida e segura entre os usuários do aplicativo, permitindo a criação de chats secretos para envio de mensagens, fotos, vídeos e arquivos de qualquer tipo, além de permitir a criação de grupos com um maior número de pessoas e bots para trabalhos compartilhados em grupos específicos (TELEGRAM, 2018).

Graças a uma infraestrutura e criptografia de múltiplos data centers, capacidade de sincronização contínua, pois os arquivos compartilhados são baseados em nuvem, e acesso às mensagens a partir de vários dispositivos de uma só vez, o Telegram permite que desenvolvedores ou qualquer pessoa com conhecimento da plataforma API botpossa criar facilmente ferramentas especializadas para atender demandas específicas.

A utilização do aplicativo surge da necessidade em se estabelecer um padrão no acionamento de inspetores, do setor de Controle da Qualidade, e de se reduzir o tempo de espera no atendimento de tarefas de manutenção classificadas como Required Inspection Item (RII) ou Itens de Inspeção Requerida (GRACIANO, 2014), que requerem acompanhamento de um inspetor na sua execução.

Tal atividade resulta em alta demanda do setor que se depara com um grande volume de acionamentos e uma demora considerável para o atendimento devido ao deslocamento, em muitos casos, desnecessários entre os hangares de manutenção.

A adoção do modelo tradicional de atendimento, até então praticado pela equipe do $C Q$, gerava para a área de manutenção um Not Value Aggregate (NVA) (ISHIKAWA, 1985), Valor Não Agregado, de $5 \mathrm{~min} / \mathrm{tec} / \mathrm{dia}$ ao longo dos turnos de trabalho representando $21 \%$ do total do NVA, dependendo da quantidade de tarefas que eram classificadas como RII e o tipo de check em 
Paulo George Miranda Martins, Rogério Aparecido Sá Ramalho, Helton Luiz dos Santos Graciano

Análise do uso do aplicativo telegram para o controle de processos de manutenção de aeronaves

questão. Neste mesmo comparativo foram utilizados os dados relativos a outras áreas de apoio que foram incluídas no projeto original, mas que não fazem parte desse objeto de estudo.

Na Figura 1 são apresentadas algumas das principais atribuições do $C Q$, no qual $90 \%$ das atividades desempenhadas concentram-se no acompanhamento de atividades na manutenção, $8 \%$ na análise de abertura de tarefas não programadas conhecidas com Non Routine Cards (NRC) ou Work Cards (WC) e $2 \%$ no auxílio a dúvidas técnicas pertinentes à interpretação de manuais de manutenção e/ou documentos técnicos normativos. Destaca-se o percentual de tempo gasto na espera por atendimento, pela manutenção, de $52 \%$, tempo empregado com apoio técnico de $31 \%$ e $17 \%$ no tempo desperdiçado pela manutenção com a procura por inspetor para atendimento de suas atividades.

Figura 1 - Demandas do Controle da Qualidade

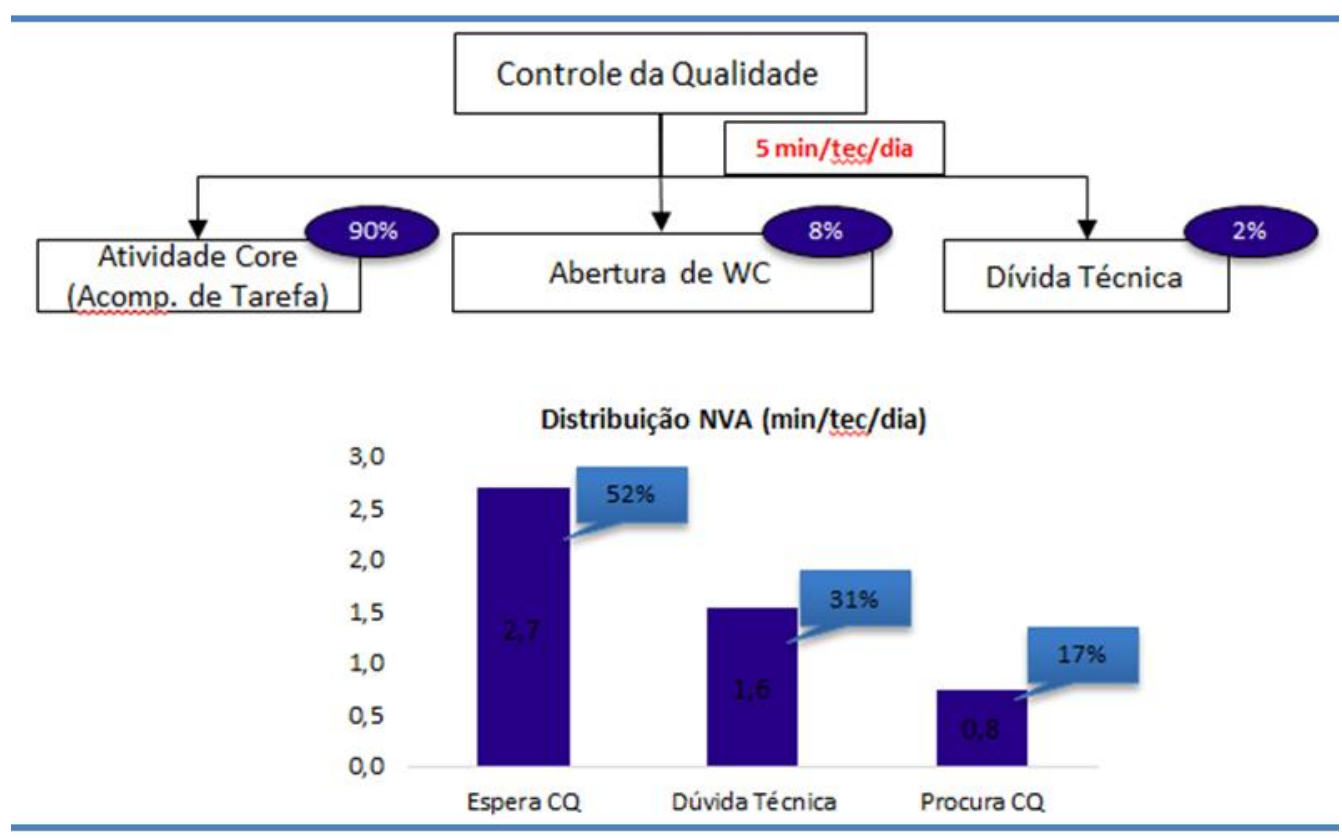

Fonte: Elaborado pelos autores.

Antes da utilização do aplicativo como ferramenta de atendimento, o acionamento dos inspetores dava-se através da solicitação via líder do departamento do $C Q$ que, dependendo da disponibilidade de sua equipe acionava um inspetor específico da área para o atendimento requerido. A grande problemática nesse processo estava no tempo de espera, pela manutenção, que 
Paulo George Miranda Martins, Rogério Aparecido Sá Ramalho, Helton Luiz dos Santos Graciano

Análise do uso do aplicativo telegram para o controle de processos de manutenção de aeronaves

estava em torno de 30 a 40 minutos para que um inspetor fosse acionado.

Outros fatores contribuintes para o tempo elevado no atendimento estava na falta de critério na priorização das tarefas a serem atendidas, falta de padronização no processo de acionamento, aeronave não configurada para a realização das inspeções e a não visualização, pelo controle da qualidade, das demandas críticas (tarefas que devem ser realizadas em um período determinado do check).

O método de Cronoanálise (AGUIAR, 2006) foi utilizado para a coleta de dados e o levantamento do tempo gasto no processo entre o acionamento pela manutenção até o pronto atendimento pela equipe do $C Q$, no qual se detectou um tempo médio de aproximadamente 30 minutos, levando-se em consideração as variáveis inerentes ao processo, quantidade de acionamentos entre as diversas aeronaves em check, chamados realizados de forma equivocada, distância entre os hangares com maior número de acionamentos, dentre outros fatores.

Nesse estudo foram considerados dados coletados e aplicáveis às três linhas e turnos de trabalho existentes no MRO durante o período de 12 dias. Foram coletados 1249 acionamentos, com $7 \%$ desse total cancelados, sendo $87 \%$ (1088) chamados direcionados à equipe do CQ e 13\% (161) direcionados à equipe de Non Destructive Test (NDT), que está subordinada à gerência de Qualidade, mas que não entra nessa pesquisa.

Testes iniciais demonstraram de pronto a eficiência da proposta que, a partir da integração do bot do aplicativo, desenvolvido especificamente para essa demanda, com o sistema MROSYs (GRACIANO, 2014) que gerencia os apontamentos de entrada e saída de mecânicos e inspetores nas tarefas de manutenção, o disparo das solicitações para o atendimento de tarefas RII seriam feitos de forma automática para todos os membros do grupo que tivessem realizado seu login de acesso ao programa incorporado no aplicativo.

A implementação e integração entre os sistemas usados nesse projeto melhorou significativamente o tempo de atendimento, levando $85 \%$ dos acionamentos para um tempo de espera menor do que 30 minutos com uma meta inicial de $90 \%$. O tempo médio de atendimento teve uma média geral de 17 
Paulo George Miranda Martins, Rogério Aparecido Sá Ramalho, Helton Luiz dos Santos Graciano

Análise do uso do aplicativo telegram para o controle de processos de manutenção de aeronaves

minutos e considerando os $90 \%$ melhores tempos, a média foi de 10 minutos. Conforme demonstrado na figura 2.

Figura 2 - Tempo médio de atendimento

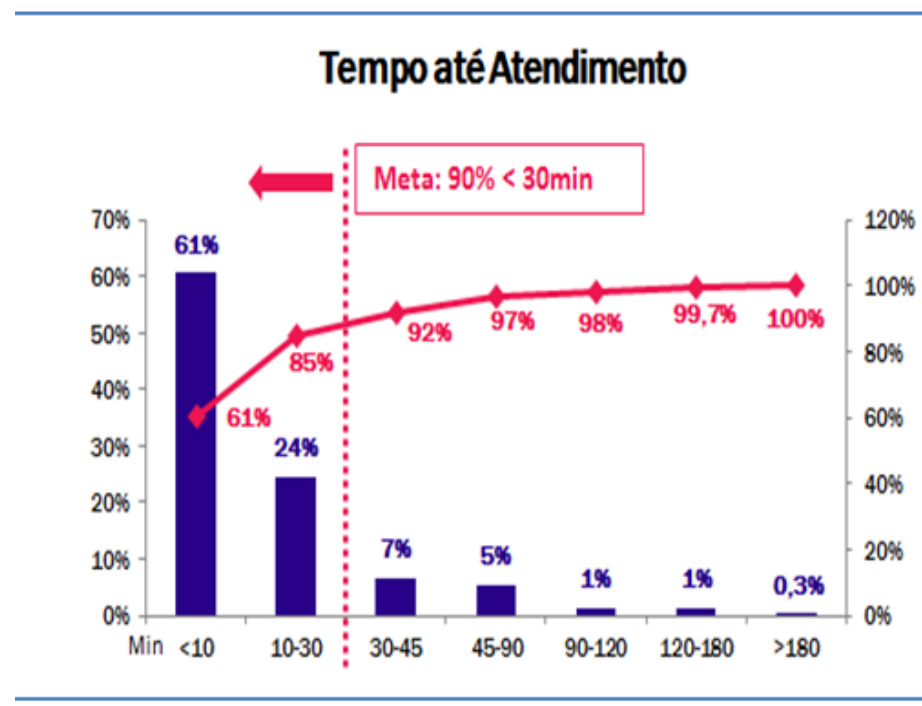

Fonte: Elaborado pelos autores.

Após a realização de vários testes ao longo dos checks que se sucederam na manutenção pelo período de um mês, estipulou-se que o tempo médio de atendimento seria em torno de 10 minutos considerando que, os requisitos préestabelecidos no projeto inicial sejam cumpridos para que não haja a incidência de retrabalho nas configurações prévias das tarefas.

Mostrando-se um modelo eficiente no planejamento de recursos para essa atividade, dado a redução significativa de tempo nos atendimentos solicitados que saíram de valor médio de 30 para 10 minutos como destacado na figura 1, o modelo proposto acabou sendo incorporado de forma permanente, sendo adicionadas ao aplicativo novas funcionalidades que contribuíram para um ganho expressivo na quantidade de tarefas atendidas por linha, pela equipe do Controle da Qualidade.

A utilização do aplicativo Telegram estabeleceu um padrão nos atendimentos do $C Q$ frente às demandas provenientes da manutenção, contribuindo para uma melhor visibilidade no detalhamento das tarefas classificadas como RII (como setup, execução e close-up das tarefas) e um gerenciamento mais eficiente das informações obtidas e dos recursos disponíveis uma vez que, tarefas consideradas críticas tem atendimento 
Paulo George Miranda Martins, Rogério Aparecido Sá Ramalho, Helton Luiz dos Santos Graciano

Análise do uso do aplicativo telegram para o controle de processos de manutenção de aeronaves

prioritário, pois, o atraso em sua conclusão pode impactar negativamente no cumprimento das demais. Ademais, estabeleceu-se uma padronização na priorização de atendimento possibilitando verificar a real necessidade de envolvimento dos inspetores nos diversos tipos de solicitações realizadas pela manutenção.

Como resultado do uso do aplicativo no atendimento das demandas é apresentado na figura 3 o fluxograma da nova tratativa de acionamento implementado nas áreas do CQ e NDT, ambos sob responsabilidade da Gerência de Qualidade, que passaram a adotar esse novo formato observando os pré-requisitos necessários para o acionamento, possibilitando um melhor direcionamento de pessoal, planejamento diário das tarefas de manutenção e adequação das demandas para as áreas do $C Q$ e manutenção e, melhor comunicação e uso de informações já que o mesmo possibilita gerar relatórios dos atendimentos realizados.

\section{Figura 3 - Fluxo de acionamentos}

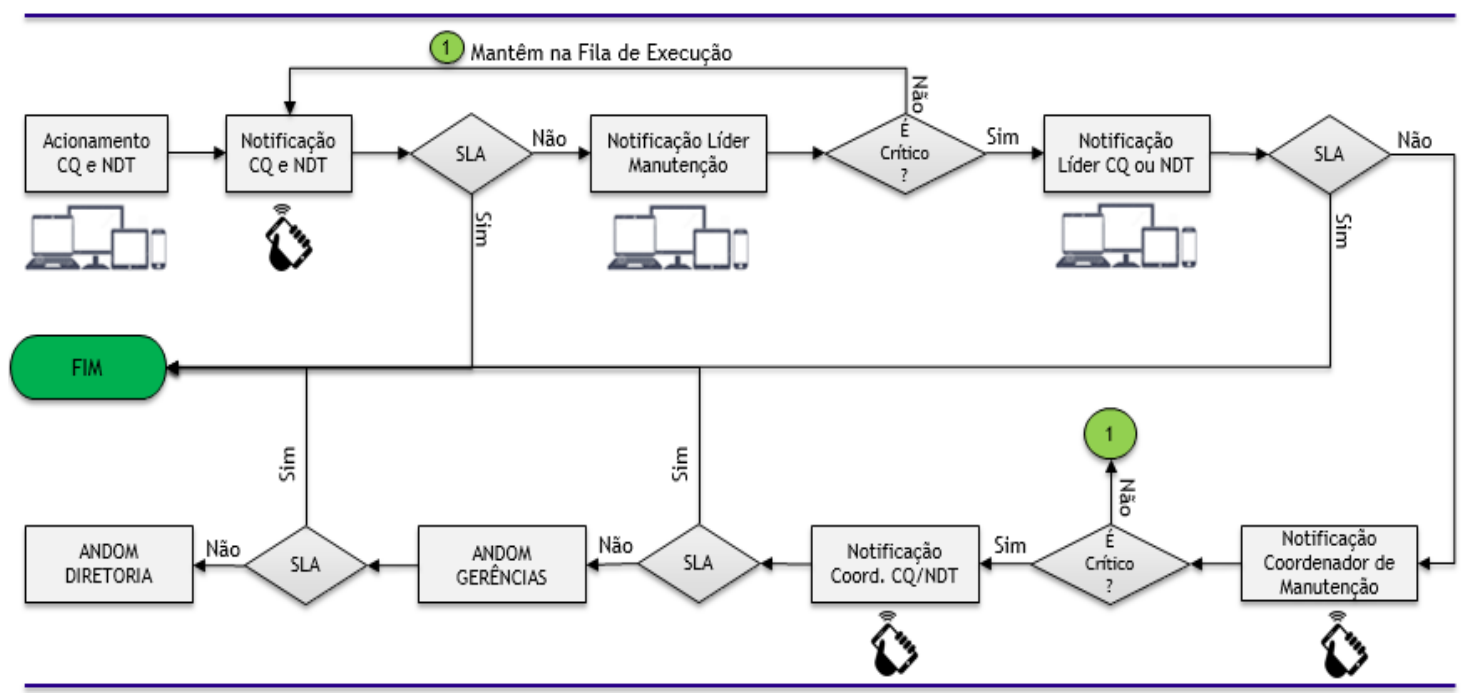

Fonte: Elaborado pelos autores.

Com essa nova estrutura de acionamentos, quando verificada a necessidade de atendimento, a manutenção faz a solicitação via sistema que imediatamente apresenta a notificação no aplicativo que, automaticamente começa a contabilizar o tempo defino para o atendimento. O Service Level Agreement (SLA) ou Tempo Limite, representa os 10 minutos necessários para o atendimento e não havendo nenhum contato por parte do $C Q$ o líder da 
Paulo George Miranda Martins, Rogério Aparecido Sá Ramalho, Helton Luiz dos Santos Graciano

Análise do uso do aplicativo telegram para o controle de processos de manutenção de aeronaves

manutenção será acionado pela equipe que realizou a solicitação e determinará se a tarefa é ou não de caráter crítico (atendimento imediato, pois compromete $o$ andamento das demais tarefas). Em caso positivo, o atendimento passa a ter prioridade sobre as demais tarefas selecionadas para área ou para a linha de manutenção como um todo.

Ressalta-se que, o ponto positivo no modelo adotado está exatamente na forma em que as equipes do $C Q$ e manutenção desempenham suas funções, gerenciam suas atividades e direcionam seus recursos, pois ao terem a seu dispor informações essenciais para o planejamento estratégico de suas demandas, podem redesenhar o caminho crítico de suas atividades e propor melhorias em seus processos.

\section{CONSIDERAÇÕES FINAIS}

O desenvolvimento de novas tecnologias presenciado nos últimos anos representa um grande avanço no setor produtivo ao dispor ferramentas capazes de proporcionar um controle mais efetivo dos processos de manutenção ou manufatura de partes. Nesse sentido, apresenta-se uma análise do uso do aplicativo Telegram para o aperfeiçoamento de processos de manutenção que busca planejar de forma eficiente o emprego de seus recursos, destacando-se a relevância do uso de novas abordagens tecnológicas para o atendimento de demandas relacionadas à Gestão do Conhecimento em ambientes corporativos.

A Gestão do Conhecimento busca, no âmbito das organizações, compreender as novas demandas de armazenamento, organização e difusão do conhecimento a partir do uso de tecnologias eficazes no gerenciamento e controle de seus processos produtivos, proporcionando uma melhor comunicação, compartilhamento de informações e aumento na sua capacidade competitiva frente às demandas internas e externas.

No setor aeronáutico, o emprego de tais tecnologias pode representar um passo importante no controle de suas atividades ao possibilitar o uso de informações precisas e selecionadas especificamente para as tomadas de decisão e promover uma melhor sinergia entre os diversos setores envolvidos diretamente ou não nos processos de manutenção. 
Paulo George Miranda Martins, Rogério Aparecido Sá Ramalho, Helton Luiz dos Santos Graciano

Análise do uso do aplicativo telegram para o controle de processos de manutenção de aeronaves

A utilização do aplicativo Telegram no controle dos atendimentos e no gerenciamento de processos internos mostrou-se uma oportunidade eficaz para o setor de Controle da Qualidade ao proporcionar uma interação sincrônica com as linhas de manutenção existentes no $\mathrm{MRO}$, permitindo maior visibilidade das demandas críticas e o emprego mais eficiente de recursos nas diversas atividades desempenhadas pela área.

Como pontos de melhoria, novas funcionalidades são incorporadas ao aplicativo visando aumentar o escopo de atendimento da equipe do $C Q$, ampliando a coleta e análise dos dados gerados a partir de relatórios provenientes dos bots criados para o aplicativo, tornando $\mathrm{o}$ atendimento $\mathrm{e}$ demais demandas atribuídas à equipe mais ágeis e sincronizadas com as propostas de segurança, qualidade, confiabilidade e entrega inerentes ao processo de manutenção de aeronaves.

Nessa perspectiva, a proposta desse estudo é apresentar um compêndio do uso das tecnologias de informação e comunicação no setor de manutenção de aeronaves, abordado nesse artigo o Telegram, tecnologias essas empregadas no contexto das novas ferramentas desenvolvidas para a chamada quarta revolução industrial que representam uma nova tendência ao empregar tais aportes no gerenciamento e controle de seus processos.

Acredita-se que, com o emprego cada vez maior dessas tecnologias em setores corporativos e de manutenção o índice de aproveitamento de recursos, dados e informações permite realizar um melhor direcionamento e controle das atividades planejadas ou não, reduzindo com isso o tempo de respostas para os desvios encontrados ao longo do processo.

\section{REFERÊNCIAS}

AGUIAR, S. Integração das ferramentas da qualidade ao PDCA e ao programa seis sigmas. Nova Lima: INDG, 2006.

ALBRECHT, K. A Terceira Revolução da Qualidade. HSM Management, v. 3, n. 17, p. 108-112, nov./dez. 1999.

BAKER, T.; BERMÈS, E.; COYKE, K.; DUNSIRE, G.; ISAAC, A.; MURRAY, P.; PANZER, M.; SCHNEIDER, J.; SINGER, R.; SUMMERS, E.; WAITES, W.; 
Paulo George Miranda Martins, Rogério Aparecido Sá Ramalho, Helton Luiz dos Santos Graciano

Análise do uso do aplicativo telegram para o controle de processos de manutenção de aeronaves

YOUNG, J.; ZENG, M. Library Linked Data Incubator Group Final Report. W3C Incubator Group Report, [online], out. 2011. Disponível em:

http://www.w3.org/2005/Incubator/lld/XGR-Ild-20111025/. Acesso em: 20 nov. 2017.

BORGES, J.; LESSA, B.; OLIVEIRA, L. O papel dos sites de redes sociais nas estratégias comunicativas de organizações da sociedade civil de SalvadorBahia-Brasil. OBS*, Lisboa, v. 8, n. 3, p. 183-203, set. 2014. Disponível em: http://www.scielo.mec.pt/scielo.php?script=sci_arttext\&pid=S164659542014000300010\&lng=pt\&nrm=iso. Acesso em: 12 abr. 2019.

CANADAY, $\mathrm{H}$. The future of MRO is human-machine teaming. Aviation Week Network, out. 2018.

CHIAVENATO, I. Introdução à teoria geral da administração. Rio de Janeiro: Elsevier, 2003.

$\mathrm{CHOO}, \mathrm{C}$. W. The knowing organization: how organizations use information to construct meaning, create knowledge, and make decisions. Library \& Information Science Research, v. 16, n. 5, p. 329-340, 1996.

CORREIA, V. M. M. O programa de manutenção de aeronaves e a sua importância na gestão da continuidade da aeronavegabilidade. 2012. 118 f. Dissertação (Mestrado em Engenharia Aeroespacial) - Instituto Superior Técnico Lisboa, Lisboa, 2012.

CRUZ, P. R. F da. Qualidade na indústria aeronáutica: aplicação da ferramenta FMEA em um setor de montagem de componentes de motores aeronáuticos. In: CONGRESSO NACIONAL DE EXCELÊNCIA EM GESTÃO \& III INOVARSE, 12., 2016, Rio de Janeiro. Anais [...]. Rio de Janeiro: FIRJAN, 2016. Disponível em: http://www.inovarse.org/node/29. Acesso em: 02 nov. 2018.

FAGUNDES, E. Contribuições de TIC para a Estratégia de Negócios. Blog. 2019. Disponível em: https://efagundes.com/artigos/contribuicoes-de-tic-para-aestrategia-de-negocios/. Acesso em: 13 abr. 2019.

FEIJO, L. P.; SILVA, N. B.; BENETTI, S. P da C. Impacto das Tecnologias de Informação e Comunicação na Técnica Psicoterápica Psicanalítica. Trends Psychol, Ribeirão Preto, v. 26, n. 3, p. 1633-1647, set. 2018. Disponível em: http://www.scielo.br/scielo.php?script=sci_arttext\&pid=S235818832018000301633\&lng=en\&nrm=iso. Acesso em: 12 abr. 2019.

GRACIANO, H. L dos S. Aplicação de uma ferramenta de business intelligence para análise das causas de variabilidade de mão de obra no contexto de manutenção de aeronaves. 2014. Trabalho de Conclusão de Curso (Especialista em Gestão da Produção) - Ciências Exatas e Tecnológicas, Universidade Federal de São Carlos, São Carlos, 2014. 
Paulo George Miranda Martins, Rogério Aparecido Sá Ramalho, Helton Luiz dos Santos Graciano

Análise do uso do aplicativo telegram para o controle de processos de manutenção de aeronaves

ISHIKAWA, K. What is total quality control? The Japanese way. Trad. David Lu. Englewood Cliffs, Prentice-Hall, 1985.

MACHADO, M. C.; URBINA, L. M. S.; ELLER, M. A. G. Manutenção Aeronáutica no Brasil: distribuição geográfica e técnica. Gestão da Produção, São Carlos, v. 22, n. 2, p. 243-253, jun. 2015. Disponível em: http://www.scielo.br/scielo.php?script=sci_arttext\&pid=S0104530X2015000200243\&Ing=en\&nrm=iso. Ācesso em: 12 nov. 2018.

MCKAY, J.; MARSHALL, P. The Dual Imperatives of Action Research. Information Technology \& People, v. 14, n. 1, p. 46-59, mar. 2001.

RAMALHO, R. A. S. Bibframe: modelo de dados interligados para bibliotecas. Informação \& Informação, Londrina, v. 21, n. 2, p. 292-306, dez. 2016. Disponível em:

http://www.uel.br/revistas/uel/index.php/informacao/article/view/26425. Acesso em: 12 fev. 2017.

SAEGER, M. M. M. T.; OLIVEIRA, M. L. P.; PINHO NETO, J. A. S.; NEVES, D. A. B. Organização, acesso e uso da informação: componentes essenciais ao processo de gestão da informação nas organizações. Páginas A\&B: Arquivos e Bibliotecas, Portugal, v. 3, n. 6, p. 52-64, 2016. Disponível em: http://hdl.handle.net/20.500.11959/brapci/69160. Acesso em: 24 jul. 2019.

SILVEIRA, R. R.; OTTANI, N.; VARVAKIS, G.; BASTOS, R. C.; FRANZONI, A. M. B. O uso da tecnologia de informação e comunicação como recurso na elaboração estratégica da informação: um estudo de caso na Gol Transportes Aéreos Ltda. In: SIMPÓSIO DE ENGENHARIA DE PRODUÇÃO, 13., 2006, Bauru. Anais [...]. Bauru: UNESP, 2006. Disponível em: http://www.simpep.feb.unesp.br/anais_simpep_aux.php?e=13. Acesso em: 20 jan. 2019.

SILVEIRA, D. T.; CÓRDOVA, F. P. A pesquisa científica. In: GERHARDT, T. E.; SILVEIRA, D. T. (Orgs.). Métodos de pesquisa. Porto Alegre: Editora da UFRGS, 2009.

SOUSA, J. L.; MARTINS, P. G. M.; RAMALHO, R. A. S. Modelos de representação semântica na era do big data. Brazilian Journal of Information Science, v. 12, n. 3, p.34-40, 2018. Disponível em: http://www2.marilia.unesp.br/revistas/index.php/bjis/index. Acesso em: 30 out. 2018.

SOUZA, L. G. S.; AGANETTE, E. C. Gestão do conhecimento em repositórios digitais: perfil das instituições brasileiras. In: ENCONTRO NACIONAL DE PESQUISA EM CIÊNCIA DA INFORMAÇÃO, 19., 2018, Londrina. Anais [...]. Londrina: UEL, 2018. p.5128-5143. Disponível em: 
Paulo George Miranda Martins, Rogério Aparecido Sá Ramalho, Helton Luiz dos Santos Graciano

Análise do uso do aplicativo telegram para o controle de processos de manutenção de aeronaves

http://enancib.marilia.unesp.br/index.php/XIXENANCIB/xixenancib/schedConf/p resentations. Acesso em: 20 jul. 2019.

TELEGRAM. Telegram FAQ. 2018. [online]. Disponível em: https://telegram.org/faq. Acesso em: 25 jul. 2019.

THIOLLENT, M. Metodologia da Pesquisa-ação. São Paulo: Cortez Editora, 2005.

\title{
ANALYSIS OF THE USE OF TELEGRAM APPLICATION TO CONTROL AIRCRAFT MAINTENANCE PROCESSES
}

\begin{abstract}
Introduction: With the development of new information and communication technologies the instant sharing of data and information has enabled significant changes in the behavior of organizations. In the field of aircraft maintenance, the use of these technologies gains new contours with the use of messaging applications in the management of their activities and control of their processes, allowing to more efficiently scale the application of resources. Objective: The main objective of this work is to analyze the use of Telegram application in the management of activities related to the Quality Control area processes of an aircraft maintenance company and its reflexes in the Knowledge Management. Methodology: It is characterized as an action research that sought to assist in the identification of guidelines and analysis of solutions for continuous improvement of processes in the area of Quality Control. Results: An analysis of the use of Telegram application to improve maintenance processes is presented, highlighting the relevance of using new technological approaches to meet the demands related to Knowledge Management in corporate environments. Conclusions: From the analysis performed it was found that the use of Telegram application contributed to a greater synergy between the work teams by sharing information essential to the process, making the service and other demands more agile and synchronized with the proposals of safety, quality and quality. reliability, favoring significant improvements in Knowledge Management processes in corporate environments.
\end{abstract}

Descriptors: Information Technologies. Process Management. Aircraft Maintenance. Innovation. Telegram.

\section{ANÁLISIS DEL USO DE LA APLICACIÓN DE TELEGRAMA PARA CONTROLAR LOS PROCESOS DE MANTENIMIENTO DE AERONAVES}

\section{RESUMEN}

Introducción: Con el desarrollo de nuevas tecnologías de información y comunicación, el intercambio instantáneo de datos e información permitió cambios significativos en el comportamiento de las organizaciones. En el campo del mantenimiento de aeronaves, el uso de estas tecnologías gana nuevos contornos con el uso de aplicaciones de 
Paulo George Miranda Martins, Rogério Aparecido Sá Ramalho, Helton Luiz dos Santos Graciano

Análise do uso do aplicativo telegram para o controle de processos de manutenção de aeronaves

mensajería en la gestión de sus actividades y el control de sus procesos, lo que permite escalar de manera más eficiente la aplicación de recursos. Objetivo: El objetivo principal de este trabajo es analizar el uso de la aplicación Telegram en la gestión de actividades relacionadas con los procesos del área de Control de Calidad de una empresa de mantenimiento de aeronaves y sus reflejos en la Gestión del Conocimiento. Metodología: Se caracteriza por ser una investigación de acción que buscó ayudar en la identificación de pautas y análisis de soluciones para la mejora continua de procesos en el área de Control de Calidad. Resultados: Se presenta un análisis del uso de la aplicación Telegram para mejorar los procesos de mantenimiento, destacando la relevancia del uso de nuevos enfoques tecnológicos para satisfacer las demandas relacionadas con la Gestión del Conocimiento en entornos corporativos. Conclusiones: Del análisis realizado se encontró que el uso de la aplicación Telegram contribuyó a una mayor sinergia entre los equipos de trabajo al compartir información esencial para el proceso, haciendo que el servicio y otras demandas sean más ágiles y sincronizadas con las propuestas de seguridad, calidad y calidad. fiabilidad, favoreciendo mejoras significativas en los procesos de Gestión del Conocimiento en entornos corporativos.

Descriptores: Tecnologías de la Información. Gestión de Procesos. Mantenimiento de Aeronaves. Innovación. Telegram.

Recebido em: 08/10/2019

Aceito em: 27/03/2020 